\title{
An Intelligent Walking Stick for the Visually-Impaired People
}

\author{
https://doi.org/10.3991/ijoe.v13i11.7565 \\ Nadia Nowshin( $\left.{ }^{\square}\right)$, Sakib Shadman, Saha Joy, Sarker Aninda, Islam Md Minhajul \\ American International University-Bangladesh (AIUB), Dhaka, Bangladesh \\ nowshineaiub.edu
}

\begin{abstract}
Blind stick or white cane is introduced to blind people after the First World War as a mobility tool to detect the obstacles in the path of the user. This paper proposes an Arduino Nano based obstacle finding stick for visuallyimpaired people, which helps a blind person by detecting the obstacles using Ultrasonic sensors and android mobile application. It is able to inform the blind person about the circumstances \& present condition of the path where he/she is walking. The main objective of this paper is to help a blind people to move more freely by using a reliable stick. The device consists of arduino nano, $\mathrm{HC}$ SR-04 ultrasonic sensor, HC-05 Bluetooth module, push buttons, 100nF ceramic capacitors and a $9 \mathrm{~V}$ battery as the power source. And the android app is developed using MIT App Inventor 2.
\end{abstract}

Keywords - White cane, Android Mobile Application, HC SR-04 ultrasonic sensor, HC-05 Bluetooth module, MIT App Inventor 2.

\section{Introduction}

According to the famous philosopher and scientist Aristotle, our knowledge about the outside world depends on five sense organs and sight is one of those five organs. And visually-impaired are those unfortunate people who are completely or partially suffering with eye sight issues. According to the survey of World Health Organization (WHO) and International Agency for Prevention of Blindness (IAPB) [1], approximately 285 million people around the world are visually -impaired among which 39 million are completely blind. Blind stick is a special device used by visually disabled people for centuries. But in recent times Electronic Travel Aids (ETAs) with sensors and sound systems are designed for improved navigation of blind people [2], [3]. Here we proposed an advanced blind stick that allows visually challenged people to navigate using advanced technology like ultrasonic sensors \& mobile application. We already know about traditional blind sticks [4]. But the question that comes into our mind is that how this system can give idea about the surrounding by using GPS system. The advantage of our project is that it can detect any obstacle with the help of ultrasonic sensors and it can provide correct location of obstacle by using the GPS system. Thus it will help blind people when they are walking outside from their home. 
Besides that if any blind people get lost then his/her relative can also know about their location by using GPS based emergency SMS system feature.

\section{Review of existing work}

ETA device has become a revolution over the last few decades to guide a visually impaired person and to make their life even easier and safer. As the ETAs getting popular day by day, researcher has been working on this field to make the device even more light, portable, safe and low cost for better service. An ultrasonic sensor based blind stick is proposed by Agarwal \& Kumar [5] in 2015 with GPS system, vibrating motor and a buzzer. As they didn't give any pictorial view of the stick, so according to device description it seems to be pretty heavy. Besides that, there was no information about how long the buzzer \& vibrator would be in ON condition. And the sending sms issue by an illiterate blind person seems to be quite impossible. Another sensor based smart stick is introduced by Gayathri in 2014[3]. It uses a GPS receiver, vibrator and a headphone to navigate the blind person. But the device has some limitations like: - water sensor can detect if the water level is over $0.5 \mathrm{~cm}$, the stick can detect only four types of obstacles (concrete wall, human body, plastic \& cardboard box) and it is not feasible to provide guidance at high intermittencies. An outdoor navigating device also came into attention in 2012[6]. The stick uses GPS technology and a SD card to store information about different locations. But the device cannot help in indoor environment as there is no GPS signal available. In 1973, an optical triangulation based laser cane was proposed, which could only detect head-height obstacles [7]. And in 1989, a navigating device equipped with a small computer and sensors was planned [7]. It took images and then translated them into a series of audio cues to inform the user which routes are blocked by obstacles. And after late nineties, wearable obstacle detection system was introduced, which was quite heavy to wear and handle [8].

\section{$3 \quad$ Proposed model}

It can be seen from Fig. 1 that there are four sensor with various attribute like A11000, B-12000,C-14000,D-15000. In our stick sensor A and B are implemented on the front side, sensor $\mathrm{C}$ on left side and senor D on right side. All four sensors detect obstacle and send the distance to an Arduino module. Then Arduino sends the correct distance to the mobile app through Bluetooth module. Then through the App user can hear the distance in their headphone. 


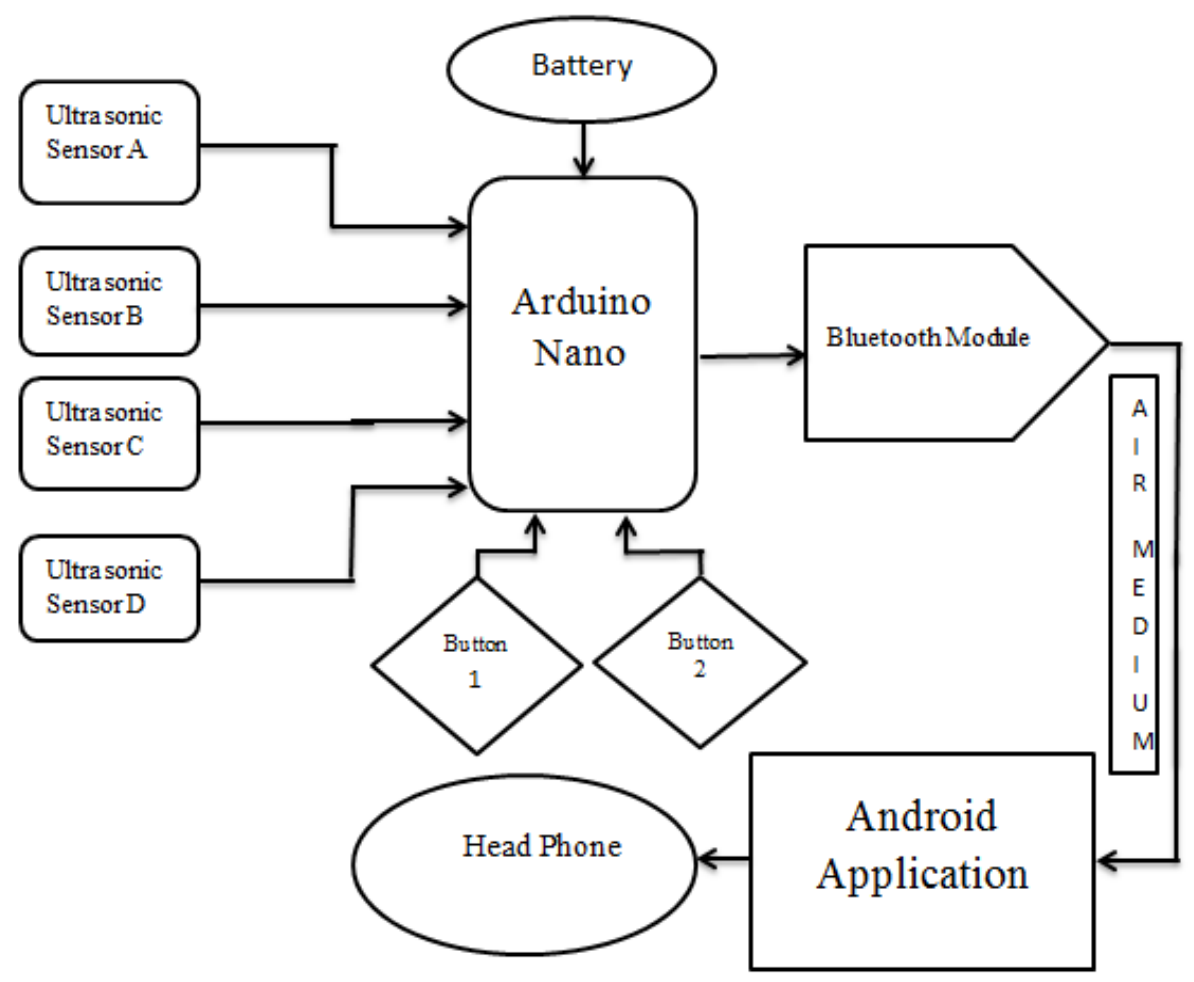

Fig. 1. Block diagram of an intelligent blind stick working process

\subsection{Software development for the movement of the walking stick}

From Fig. 2 it can be seen that for the software simulation the chosen build-in components are arduino nano, ultrasonic sensor HC SR-04, virtual terminal as Bluetooth module, $100 \mathrm{~K}$ variable resistors for setting the range of ultrasonic sensor, push button, $100 \mathrm{nf}$ capacitor for hardware denouncing [2]. As Proteus Professional v8 SP2 does not offer any specific ultrasonic sensor device, so here we used a variable resistor as sensor controller. When it is connected through a mobile phone Bluetooth application, it provides a baud rate of 9600 . The echo and trigger pins of the ultrasonic sensors are connected to arduino pin D4, D6, D8, D10 and D5, D7, D9, D11 respectively. The Tx pin of the arduino is connected to Rx pin of the Bluetooth module and Rx pin is connected to Tx pin. Two push buttons are connected to two interrupt enabled pin D2 and D3. Proteus was used to check the circuitry and the sensor outputs are shown via virtual terminal. And Fig.3 illustrations the virtual terminal results like- 12426, 12246, $12235,15105,14325$ etc. Here the first two digits represents the sensor number like "12" (for sensor B) and the next three digits " 426 " represents the obstacle distance in centimeters. 


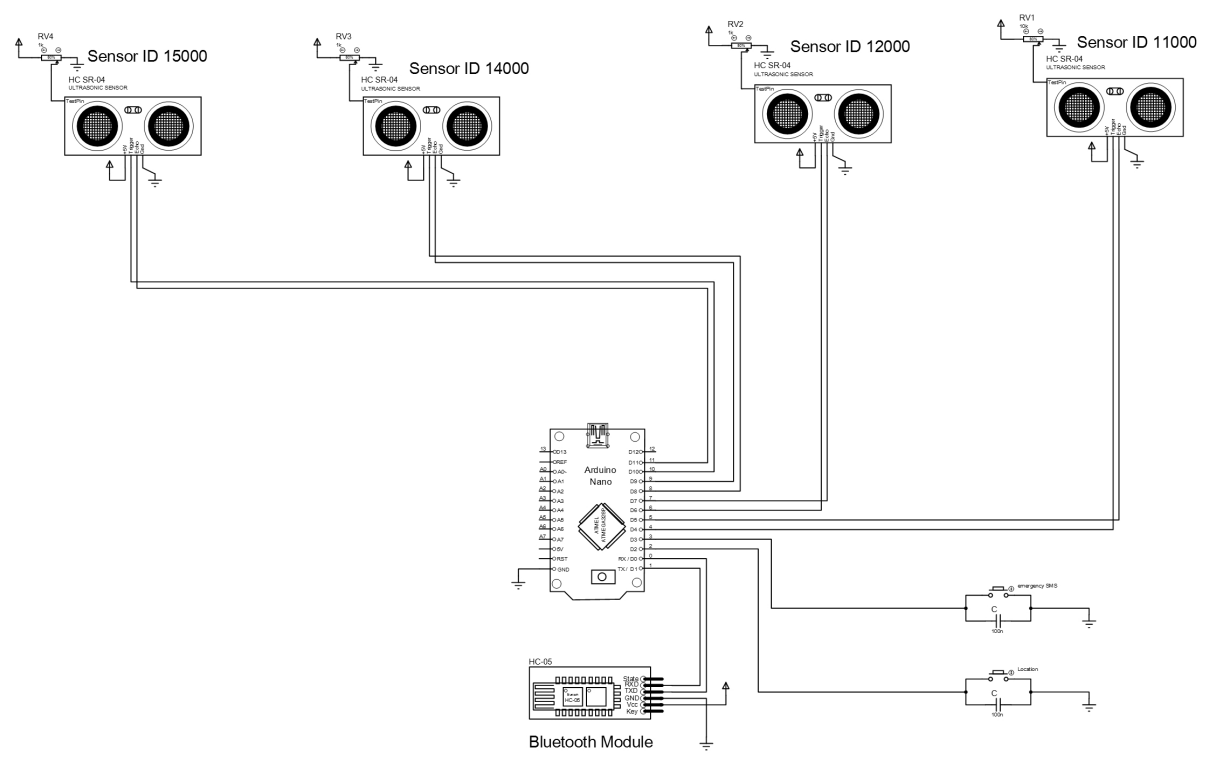

Fig. 2. Simulation of the blind stick via Proteus 8.0

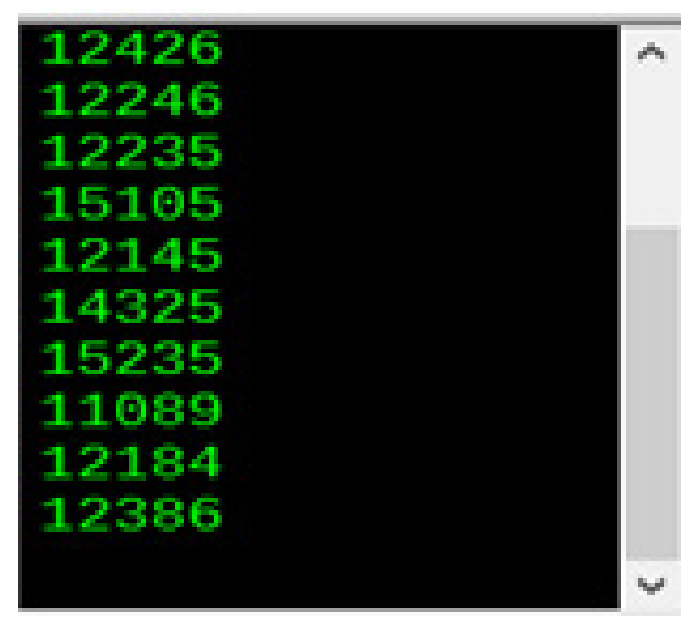

Fig. 3. Simulation of the blind stick via Proteus 8.0

\subsection{Hardware development for two push button connection}

In the proposed model two push buttons are used as shown in Fig.5, one for sending the emergency SMS to a predefined phone number and the other one is for getting the current location of the user. The buttons are connected to two interrupt enabled pins of the Arduino - D2 and D3. 100nF ceramic capacitor is connected in parallel with each push button for hardware debouncing purpose. 


\subsection{Hardware development for Bluetooth module}

In our white stick, HC-05 Bluetooth module is used for receiving and processing the information received from the Arduino board. And it can be seen from Fig. 5 that the TX \& RX pins of Bluetooth module is connected to the TX \& RX pins of Arduino respectively. Universal Asynchronous Receiver/Transmitter (UART) protocol is used for transmitting and receiving data signal.

\section{Overall working principle of the walking stick}

Fig.6 portrays the overall view of the blind stick. The stick consists of eight ultrasonic sensors at the front side to cover the surrounded area (front, left and right side) of a visually impaired person and the sensors detected distance of the obstacle is send to the user headphone via a mobile app.

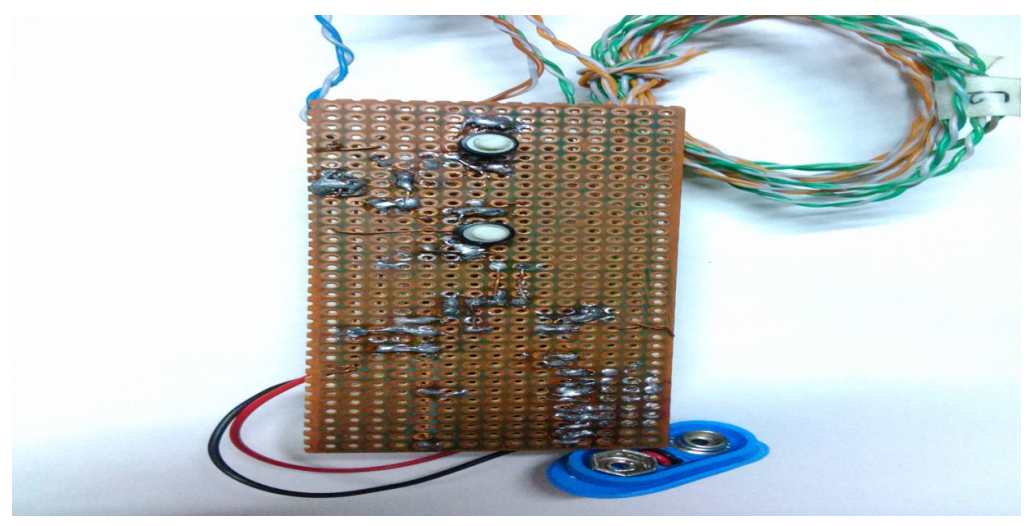

Fig. 4. Push button connection of the stick

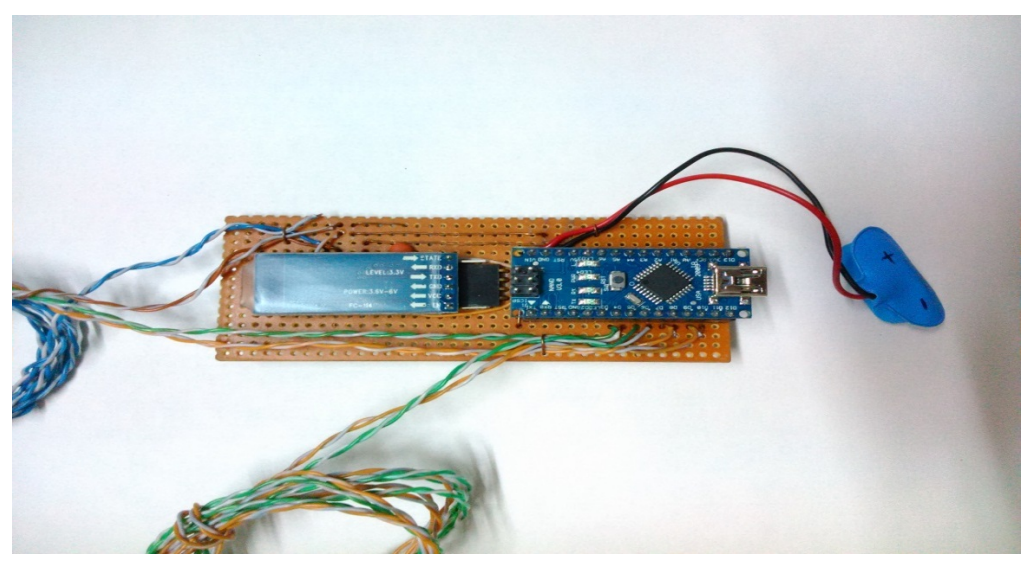

Fig. 5. Bluetooth module connection of the stick 


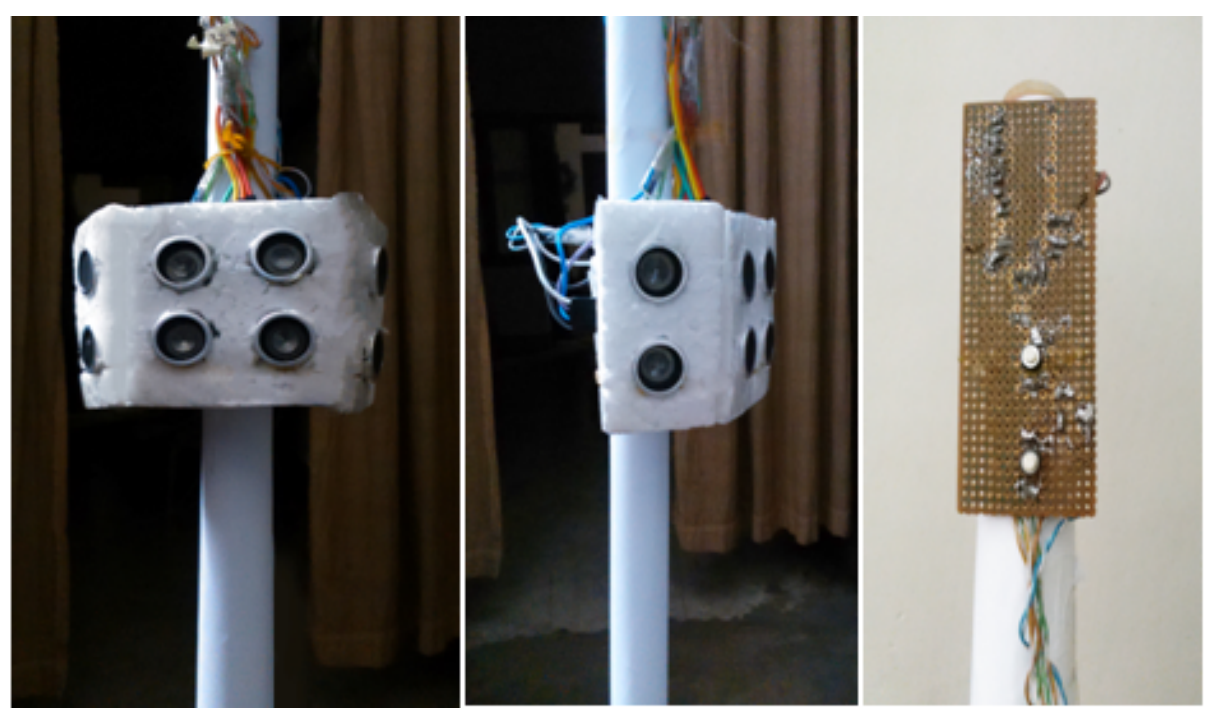

Fig. 6. Blind stick (front view, side view \& back view)

When any of the four sensors gets a new obstacle in it's path than it will be automatically updated into mobile app. Here the Arduino Nano used as the main controller for RX and TX pin of Bluetooth device. UART communication protocol is used to transmit data signals and a $9 \mathrm{~V}$ li-lion battery is used as a power supply. Here we also used two push buttons at the handle of the stick with a capacitor and both of them are connected to the Arduino interrupt pin .One button is used for the emergency text transmission purpose from the user and second one is used to know self-location of the visually challenged person.

In our model an android phone is used for the mobile app[9] purpose and the phone is connected to Arduino through Bluetooth module [3] . The sound processing is done by the Arduino Nano, which can send data (obstacle distance) to the user mobile with the help of an android application [10] through a Bluetooth module via UART communication protocol. Here the user can hear the obstacle distance as a voice massage from the android phone.

One more purpose of this application software is that it can send a emergency text message to two /three family members of the blind person containing the location detail of the blind person, if he/she gets lost.

Fig.7 shows the setting option of the mobile application through which the threshold distance of the obstacle can and any three emergency contact numbers can be predefined. So that if the visually impaired person gets lost then he/she can send his/her current location through a push button via this app. 
settings Screen

Current Settings:

Threshold Distance: $30 \mathrm{~cm}$.

Emergency Text Number: 01738743444

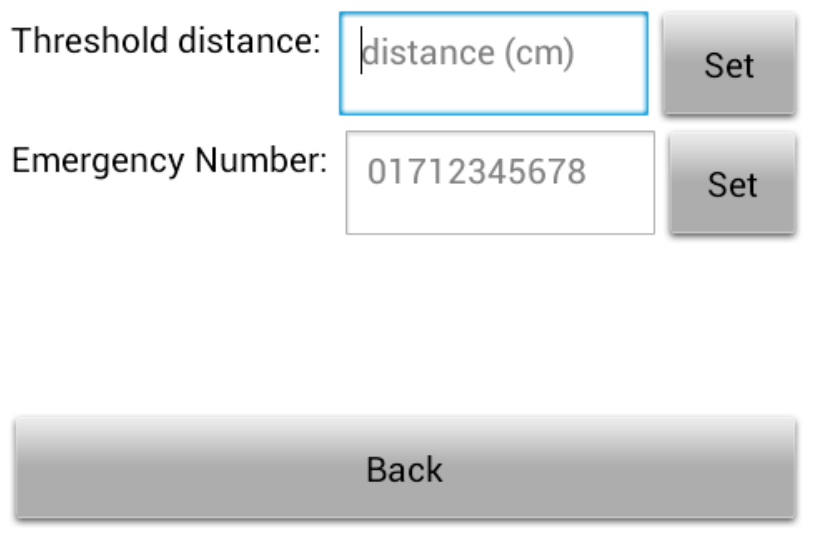

Fig. 7. Output from the Android mobile applications

\section{$5 \quad$ Future scope and conclusion}

The technologies behind blind sticks are upgrading day by day. And our model ensures one thing that is making the task of moving of a blind person easy and comfortable. The stick is also very light and handy to carry. And the components or parts that we used in the stick are also easily available and less in cost. And besides all that the manufacturing cost is also quite low, that makes the stick affordable for people of all class and age. In future, if further improvement and investment is carried out with the stick then it will be an even more effective device for the future world.

Some of the techniques in which this device can be modified are given below:

- Arduino can be replaced by upgraded Microcontroller or chip.

- It can be further enhanced by using VLSI technology to design the PCB unit. This can make the system even more compact.

- More sensors can be used for further application.

- Image processing can be used for knowing about the volume of obstacles and object patterns.

- GPS tracker can be used for more accurate location.

- Android application can be developed.

- High range ultrasonic sensor can be used. 


\section{References}

[1] World Health Organization, "Universal eye health: a global action plan 2014-2019"World Health Organization 2014.

[2] Rene Farcy, Roger Leroux, Alain Jucha, Ronald Damaschini, Colette Gregoire, Aziz Zogaghi " Electronic Travel Aids And Electronic Orientation Aids For blind people: Technical, Rehabilitation And Everyday Life Points Of View", Conference \& Workshop on Assistive Technologies for People with Vision \& Hearing Impairments Technology for Inclusion CVHI 2006.

[3] Gayathri, G., Vishnupriya, M., Nandhini, R., and Banupriya, M. M."SMART WALKING STICK FOR VISUALLY IMPAIRED.” International Journal Of Engineering And Computer Science, Vol.3, pp.4057-4061,2014.

[4] K. Chaitrali, D. Yogita, K. Snehal, D. Swati, and D. Aarti, "An intelligent walking stick for the blind," International Journal of Engineering Research and General Science, vol. 3, Issue1, November, 2016.

[5] Ankit Agarwal, Deepak Kumar, Abhishek Bhardwaj "Ultrasonic Stick for Blind," International Journal of Engineering and Computer Science, vol. 4, Issue 4, April, 2015.

[6] Anon, n.d. Electronic Walking Stick for the Blind, s. 1.:s.n.

[7] Johann Borenstein, and Iwan Ulrich "The Guide Cane-A Computerized Travel Aid for The Active Guidance Of Blind Pedestrians," IEEE International Conference on Robotics and Automation, Albuquerque, NM, Apr.21-27, 1997.

[8] Sylvain Cardin, Daniel Thalmann and Frederic Vexo, "Wearable Obstacle Detection System for visually impaired People".

[9] "Debouncing contacts and switches in embedded systems,". [Online]. Available: http://www.ganssle.com/debouncing.htm. Accessed: Nov. 1. 2016.

[10] ]"Debouncing contacts and switches in embedded systems,". [Online]. Available: http://www.ganssle.com/debouncing.htm. Accessed: Nov. 1. 2016.

\section{Authors}

Nadia Nowshin completed Masters in Telecommunication Engineering and B.Sc in Computer Engineering from American International University-Bangladesh(AIUB) $\&$ also working as an Assistant Professor in the Department of EEE in AIUB. Her research interest includes Nano technology, embedded system design, signal processing and opto-electronics.

Shadman Sakib completed his graduation in EEE from American International University-Bangladesh. He has completed his school from PC Nall Memorial High School. He is interested in computer vision machine learning.

Joy Saha completed his graduation in EEE from American International University-Bangladesh \& also a member of IEEE. He completed his S.S.C from Kishoreganj Govt Boys' High School \& H.S.C from Cambrian College.

Aninda Sarker completed his graduation in EEE from American International University-Bangladesh He is interested in Robotics \& Nano technology.

Md Minhajul Islam completed his graduation in EEE from American International University-Bangladesh. He completed his S.S.C from Cantonment public school and college Rangpur and HSC from Rangpur Government college.

Article submitted 09 August 2017. Published as resubmitted by the authors 17 September 2017. 